\title{
The Usefulness of The Ultrasound Diagnosis of Ovarian Masses Based on The O-RADS Classification System
}

\author{
Hoda Abdel Kareem Ahmed ${ }^{1, *}$ MD. and Faisal Ali Mustafa ${ }^{2}$ MD.
}

\author{
* Corresponding Author: \\ Hoda Abdel Kareem Ahmed \\ hodaradiology2012@gmail.com
}

Received for publication August 28 2021; Accepted October 01, 2021, Published online October 01, 2021.

Copyright The Authors published by Al-Azhar University, Faculty of Medicine, Cairo, Egypt. Users have the right to read, download, copy, distribute, print, search, or link to the full texts of articles under the following conditions: Creative Commons Attribution-Share Alike 4.O International Public License (CC $B Y-S A$ 4.0)

doi: 10.21608/aimj.2021.90943.1551

${ }^{I}$ Radio-diagnosis Department, Faculty of Medicine, Al-Azhar University Assuit, Egypt.

${ }^{2}$ Obestretric.-Gyna Department, Faculty of Medicine, Al-Azhar University Assuit, Egypt.

\begin{abstract}
Aim of the work: Ovarian malignancies represent the leading cause of mortality from gynecologic cancers and its diagnosis is a special challenge. Ultrasonography is the primary imaging modality and the Ovarian-Adnexal Reporting and Data System (O-RADS) US was made to provide consistent interpretations that can help in management recommendation. The present study aimed to evaluate the usefulness of using the O-RADS classification system US in the diagnosis of suspicious ovarian masse lesions.

Patients and methods: Fifty patients " 15 pre and 35 post-menopausal" with suspicious adnexal mass lesions, their ages ranged from 19-67 years $(\mathrm{M}=41.8 \pm 3.64$ years). Trans-abdominal \& trans-vaginal US with Color/Power Doppler examination were done to all patients. The US ORADS classification system was used for evaluation of the all studied adnexal mass lesions.

Results: Lesion morphology including size, consistency and vascularity were analyzed and scored using the US O-RADS system with selected proper line of management revealed, 13 lesions as O-RADS3 (likely benign) while 18 lesions scored as O-RADS4 \&19 lesions scored as ORADS5 (likely malignant). The O-RADS scoring system showed a high sensitivity $94.12 \%$, specificity $68.75 \%$, and accuracy $86 \%$ with $86.49 \%$ PPV \& 84.62 NPV.

Conclusion: The U/S O-RADS classification system was found to be a valuable non-invasive diagnostic tool of suspicious ovarian masses with high sensitivity in differentiation between benign and malignant neoplastic lesions.
\end{abstract}

Keywords: Ultrasound; Suspicious ovarian lesions, O-RADS.

Disclosure: The authors have no financial interest to declare in relation to the content of this article. The Article Processing Charge was paid for by the authors.

Authorship: All authors have a substantial contribution to the article.

\section{INTRODUCTION}

Ovarian malignancy represents the leading cause of death from gynecologic cancers with its greatest risk occurring after menopause. ${ }^{1}$ The adnexal lesions can be of functional etiology, inflammatory changes, benign and malignant neoplasm. ${ }^{2}$

Adnexal masses are common health problem that lead to clinical workload that need diagnostic imaging, surgery, and pathology. ${ }^{3}$ The characterization of ovarian mass lesions is significant both to decrease unnecessary anxiety and enable decisions regarding optimal management. ${ }^{4}$ Adnexal mass diagnosis has a special challenge owing to that benign adnexal masses greatly outnumber the malignant ones and to determine the degree of malignancy suspicion is critical which is mainly based on imaging appearance. ${ }^{5}$

Ultrasound (US) is a simple and noninvasive diagnostic method that considered the primary imaging modality for identifying and characterizing of ovarian masses. ${ }^{6}$ The US Color Doppler findings 


\section{PATIENTS AND METHODS}

Patients: This prospective study included 50 patients, 15 pre and 35 post-menopausal who referred to diagnostic ultrasonography unite for assessing a suspicious adnexal mass lesion. Their ages were 1967 years $(M=41.8 \pm 3.64)$. Study ethics committee approvals were obtained for this work.

Inclusions criteria: any patient had a suspicious adnexal mass lesion

Exclusions criteria: patients with O-RADS score1-2 classic form. Also patients who could not tolerate full bladder and whom did not come for follow up or surgical interference.
U/S protocol and technique:-

Either trans-abdominal US examination with fully distended urinary bladder or trans-vaginal US exam. After UB evacuation was done using Semen's Acuson x300 machine with transducer frequencies ranged from 2.5-8 $\mathrm{MHz}$ while patient lying supine and multi-directional sonograms were taken through the area of interest. Each adnexal lesion was categorized according to its:-size, location, internal consistency, and definition of borders. Color or power Doppler US was used to assess lesion vascularity and to ensure if there is any solid component. Each lesion was evaluated and scored based on the US O-RADS classification system , table I. ${ }^{10}$

\begin{tabular}{|c|c|c|}
\hline $\begin{array}{l}\text { O-RAD } \\
\text { US score }\end{array}$ & $\begin{array}{c}\text { Risk of } \\
\text { malignancy }\end{array}$ & Descriptors \\
\hline 3 & $\begin{array}{c}1 \text { to }<10 \% \\
\%\end{array}$ & $\begin{array}{c}\text { Mature teratoma/hemorrhagic cyst/endometrioma } \geq 10 \mathrm{~cm} \\
\text { Unilocular cyst with irregular inner wall }(<3 \mathrm{~mm} \text { height }) \\
\text { Multilocular cyst }<10 \mathrm{~cm} \text { with smooth inner wall, CS } 1-3 \\
\text { Solid smooth lesion, any size, CS } 1\end{array}$ \\
\hline 4 & 10 to $<50 \%$ & $\begin{array}{c}\text { Unilocular cyst with solid component ( } 0-3 \text { papillary projections) } \\
\text { Multilocular cyst } \\
\geq 10 \mathrm{~cm} \text { with smooth inner wall, CS } 1-3 \\
\text { Any size with smooth inner wall, CS } 4 \\
\text { Any size with irregular inner wall and/or septation, any CS } \\
\text { With solid component, CS } 1-2 \\
\text { Solid smooth lesion, any size, CS } 2-3\end{array}$ \\
\hline 5 & $\geq 50 \%$ & $\begin{array}{c}\text { Unilocular cyst with } \geq 4 \text { papillary projections, any CS } \\
\text { Multilocular cyst with solid component, CS 3-4 } \\
\text { Solid smooth lesion, CS } 4 \\
\text { Solid irregular lesion, any CS } \\
\text { Peritoneal findings (ascites or nodules) }\end{array}$ \\
\hline
\end{tabular}

Table 1: O-RADS, US scores, 3-5 .

Histo-pathology and final clinical diagnosis as a reference standard:-The US findings with the ORADS classification system were correlated by surgical excision and pathology results for 39 suspicious masses where the remaining 11 benign featuring lesions in young pre-menopausal patients were followed up for a period of 6-12 months until reaching the final clinical diagnosis.

Statistical analysis:-

The statistical calculations were done using Statistical Package for the Social Science in which data described in terms of range, mean \pm standard deviation, $(\mathrm{M} \pm \mathrm{SD})$, percentages. Sensitivity, specificity, accuracy, positive predictive value and negative predictive value for US O-RADS results for ovarian masses.

\section{RESULTS}

This study included 50 patients with 50 suspicious ovarian mass lesions. Fifteen patients were premenopausal and 35 were post-menopause. Most postmenopausal patients were asymptomatic (29/35) while pain was the main complaint in the premenopausal patients (13/15), Table II.

\begin{tabular}{cccc}
\hline C/O & $\begin{array}{c}\text { Pre- } \\
\text { menopausal }\end{array}$ & $\begin{array}{c}\text { Post- } \\
\text { menopausal }\end{array}$ & Total \\
\hline Asymptomatic & 3 & 29 & 31 \\
Pain & 13 & 5 & 18 \\
\hline Vaginal discharge & 5 & 2 & 7 \\
Bleeding & 5 & 1 & 6 \\
Pelvic mass & 3 & - & 3 \\
Diarrhea/constipation & 1 & 2 & 3 \\
Amenorrhea & 2 & - & 2 \\
\hline
\end{tabular}

Table 2: pt. demographic data and $\mathrm{C} / \mathrm{P}$ in all patients $(\mathrm{no}=50)$

US O-RADS diagnosis results of studied 50 adnexal lesions:-

Lesion morphology including size, consistency and vascularity were analyzed and scored using the U/S O-RADS scoring system with selected proper line of management. Thirteen lesions were scored as $\mathrm{O}$ RADS 3 which considered likely of benign etiology while 18 lesions scored as O-RADS 4 and 19 lesions scored as O-RADS 5 that considered likely of malignant etiology according to the imaging findings. Table III. 


\begin{tabular}{|c|c|c|c|c|c|}
\hline \multicolumn{3}{|l|}{ US Lesion Morphology } & \multirow{2}{*}{$\mathrm{CD}$} & \multirow{2}{*}{ O-score } & \multirow{2}{*}{ Management } \\
\hline texture & size & no & & & \\
\hline Mature teratoma, & $10.5 \mathrm{~cm}$ & 1 & - & 3 & Surgery \\
\hline Hemorrhagic cyst & $11-13 \mathrm{~cm}$ & 3 & - & 3 & $\begin{array}{l}2 \text { Follow up } \\
1 \text { Surgery }\end{array}$ \\
\hline $\begin{array}{l}\text { Unilocular cyst with irregular inner wall < } \\
\qquad 3 \mathrm{~mm} \text { height }\end{array}$ & $4-8 \mathrm{~cm}$ & 4 & CS $3 / 4$ & 3 & $\begin{array}{l}2 \text { follow up } \\
\& 2 \text { Surgery }\end{array}$ \\
\hline $\begin{array}{l}\text { Multilocular cyst with smooth inner } \\
\text { wall/septae }\end{array}$ & $<10 \mathrm{~cm}$ & 4 & CS $1-3$ & 3 & $\begin{array}{l}3 \text { follow up } \\
\& 1 \text { surgery }\end{array}$ \\
\hline Solid with smooth outer & $7 \times 6 \mathrm{~cm}$ & 1 & CS 1 & 3 & surgery \\
\hline $\begin{array}{c}\text { Unilocular cyst with solid component }(0-3 \\
\text { papillary projections }\end{array}$ & $5-7 \mathrm{~cm}$ & 2 & - & 4 & $\begin{array}{l}1 \text { follow up \& } 1 \\
\text { surgery }\end{array}$ \\
\hline Multilocular cyst with smooth inner wall & $\geq 10 \mathrm{~cm}$ & 6 & -CS $1-3$ & 4 & Surgery \\
\hline Cyst with thick smooth inner wall & $6-11 \mathrm{~cm}$ & 5 & CS 4 & 4 & $\begin{array}{l}2 \text { follow up } \\
\& 3 \text { surgery }\end{array}$ \\
\hline $\begin{array}{c}\text { cyst with irregular inner wall and/or } \\
\text { septation, }\end{array}$ & $5-13 \mathrm{~cm}$ & 5 & CS $1-2$ & 4 & Surgery \\
\hline $\begin{array}{c}\text { Unilocular cyst with } \geq 4 \text { papillary } \\
\text { projections }\end{array}$ & $4-9 \mathrm{~cm}$ & 3 & $\begin{array}{c}- \\
\text { any }\end{array}$ & 5 & Surgery \\
\hline Multilocular cyst with solid component & $8-14 \mathrm{~cm}$ & 4 & CS 3-4 & 5 & Surgery \\
\hline Solid smooth lesion & $8 \times 6 \mathrm{~cm}$ & 1 & CS 4 & 5 & Surgery \\
\hline Solid irregular & $6-15 \mathrm{~cm}$ & 7 & CS3 & 5 & Surgery \\
\hline+ peritoneal findings & $8-15 \mathrm{~cm}$ & 4 & CS3-4 & 5 & surgery \\
\hline
\end{tabular}

Table 3: Lesion US morphology with US O-RADS score 3-5 diagnosis of studied lesions (no=50)

Histopathology and final clinical diagnosis results of the studied 50 adnexal lesions:-

Our reference standard was the histopathology results for 39 surgical excised lesions while 11 lesions undergo follow up for 6- 12 months until reaching the final clinical diagnosis. Malignant lesions represented by $70 \%$ of studied lesions $(35 / 50)$ with Mucinous cystadenocarcinoooma found to be the commonest pathology (36\%) followed by Serous cyst-adenocarcinoma (32\%) in the other hand , 15/50

\begin{tabular}{cccc}
\hline Lesion final (Histopathology/clinical) & Pt. age & Percent $\%$ \\
\hline diagnosis & \& Menopause sate & No. & $30 \%$ \\
Benign lesions:- & $19-47$ yes (Pre-M.) & 15 & $12 \%$ \\
Follicular cyst & 6 & $8 \%$ \\
Hemorrhagic cyst & 4 & $6 \%$ \\
Fibroma & 3 & $2 \%$ \\
Teratoma & $33 \& \geq 50 y s$ (1Pre/34 Post M) & 35 & $70 \%$ \\
Malignant lesions:- & 1 & $36 \%$ \\
Mucinous cystadenocarcinoooma & & 18 & $32 \%$ \\
Serous cyst-adenocarcinoma & & 16 & $2 \%$ \\
Endometriod carcinoma & & 1 & $2 \%$ \\
\hline
\end{tabular}

Table 4: Histopathology \& final clinical diagnosis results of the studied 50 adexeal lesions $($ no $=50)$.

Analysis of the lesion US O-RADS score in correlation with the final diagnosis of studied 50 adnexal lesions:-

According to the O-RADS score classification system in correlations to the reference standard until reaching the final diagnosis, 11 lesions were true negative and 32 were true positive while 5 lesions were false positive and 2 lesions were false negative, Table V. Fig.,1-6 lesions (30\% ) found to be of benign etiology with Cystadenoma-cystadenofibroma, either serous/mucinous found to be the commonest benign pathology $(12 \%)$ followed by Follicular cyst $(8 \%)$. There is only 1 fibroma lesion that misdiagnosed by the U/S O-RADS as a malignant lesion,. Malignancy etiology was the most prevalent pathology in elderly post-menopausal patients, 34 lesions while the all benign "15 lesions" were detected in young premenopausal women. Table IV. 


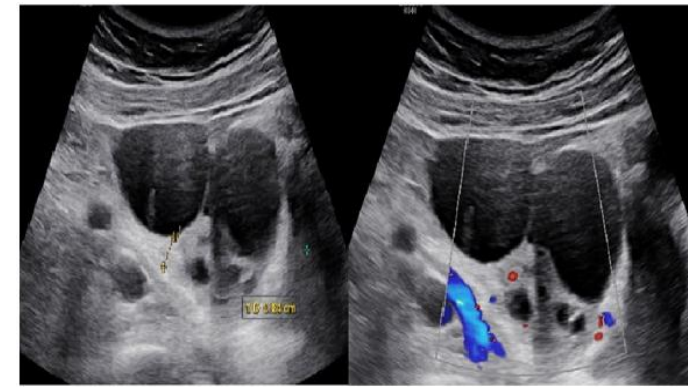

Fig. 1: A young female aged 29 years complaining of pelvic pain: a) Abd. U/S exam. revealed a sizable multi-locular cystic lesion $<10 \mathrm{~cm}$ with smooth inner wall b) Color Doppler exam. Showed no vascularity (CS1), O-RADS 3 (Benign featuring cystic lesion). Histopathology: Serous cystadenoma.

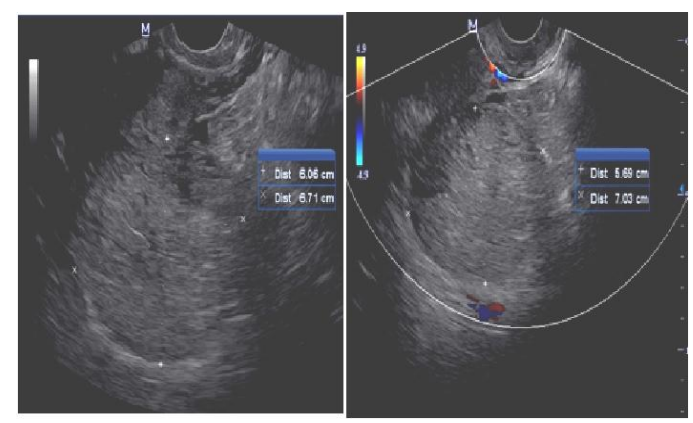

Fig. 2: A young female aged 22years with irregular vaginal bleeding a) TV U/S exam. showed a well demarcated solid lesion with smooth outlines. b) Color Doppler exam. showed no vascularity (C1), ORADS 3. (Benign featuring ovarian cystic lesion). Histopathology: Fibroma.

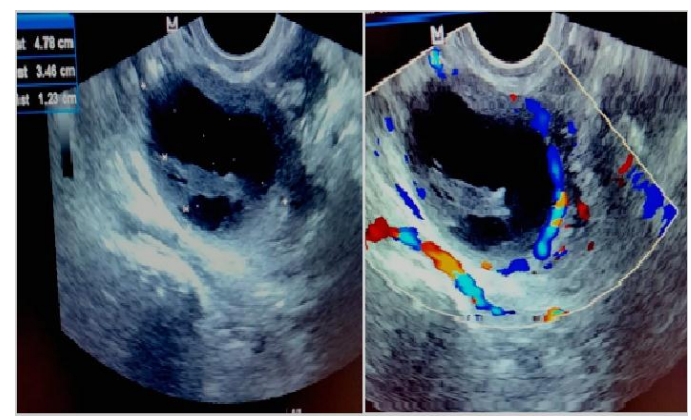

Fig. 3: Middle aged female (42 years) with history of vaginal discharge: a) TV U/S exam. revealed a unilocular cystic lesion with irregular inner walls and papillary projection. b) Color Doppler exam. showed moderate vascularity(C3) O-RADS 4 (Malignant featuring ovarian mass lesion). Histopathology: Mucinous cystadenoma.

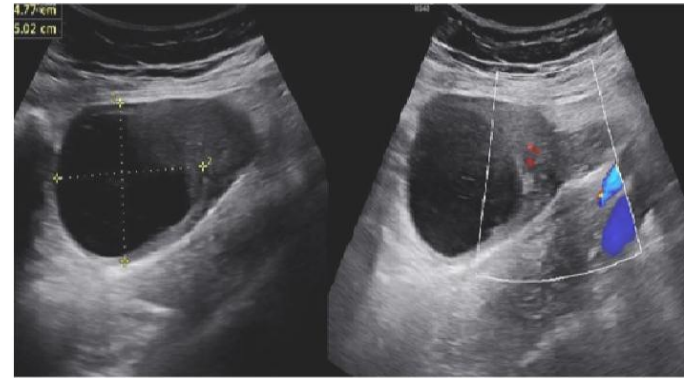

Fig. 4: Asymptomatic female aged 34 years a) Abd. U/S exam. revealed a unilocular cyst with smooth outlines and a solid component .b) Color Doppler exam. Showed minimal vascularity (C2), O-RADS 4. (Benign featuring cystic lesion). Histopathology: Endometriod ovarian carcinoma.

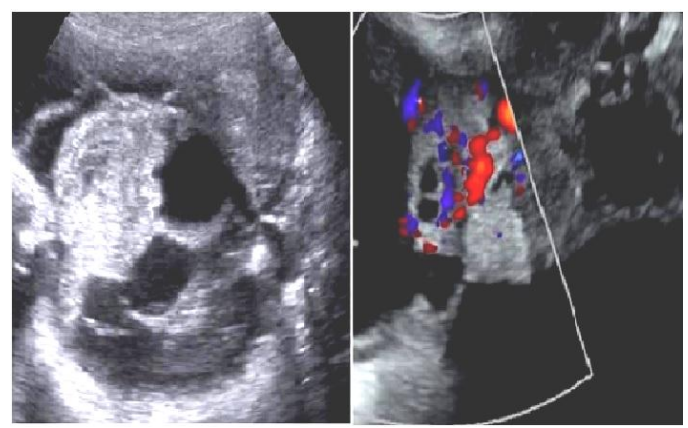

Fig. 5: An elderly female aged 60 years with history of pelvic pain: a) Abd. U/S exam. revealed a sizable multi-locular cystic lesion with solid component and ascites b) Color Doppler exam. showed marked vascularity (C4) O-RADS 5 (Malignant featuring mass lesion). Histopathology: Mucinous cystAdenocarcinoma.

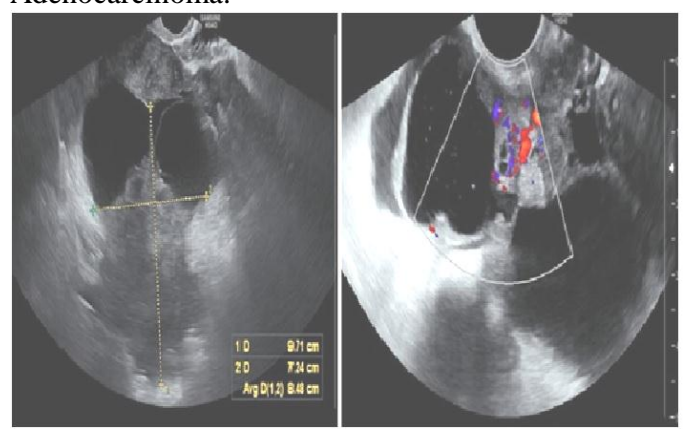

Fig. 6: A female aged 51 years with history of pelvic pain: a)TV U/S exam. revealed a sizable multilocular cystic lesion with solid component, b) Color Doppler exam. showed moderate vascularity(C3) ORADS 5 (Malignant featuring mass lesion). Histopathology: serous cyst-Adenocarcinoma.

Statistical results of US O-RADS in the diagnosis of the studied 50 suspicious adnexal masses:-

Applying the O-RADS scoring system to the studied 50 suspicious adnexal lesions with the histopathology $\&$ follow up clinical diagnosis as a reference standard; It showed a high sensitivity $94.12 \%$, specificity $68.75 \%$, and accuracy $86 \%$ with $86.49 \%$ positive predictive value $\& 84.62$ negative predictive value, Table VI. 


\begin{tabular}{cccccc}
\hline & Sensitivity & Specificity & Accuracy & PPV & NPP \\
\hline US/O-RADS & $94.12 \%$ & $68.75 \% \%$ & $86 \%$ & $86.49 \%$ & $84.62 \%$ \\
\hline
\end{tabular}

Table 6: Statistical results of US O-RADS in the diagnosis of the studied 50 suspicious adnexal masses.

\section{DISCUSSION}

Cancer Ovary showed a high case-fatality ratio with its diagnosis often presents diagnostic and management dilemmas. ${ }^{11,12}$

Our study included 50 patients with suspicion ovarian masses; the majority of them were asymptomatic while the pain was the main complaint in some patients. This was in agreement with Dipak $\mathrm{AB}$ et al 2009, who stated that many adnexal masses are asymptomatic while abdominal pain was seen in about $92 \%$ of patients, ${ }^{13}$ Also, Givens V et al 2009 said that:- Pelvic or abdominal pain was the predominate symptoms reported by women with ovarian cancer. ${ }^{1}$ In the other hand, Ebell $\mathrm{MH}$ et al 2016 \& Goff BA et al 2004 stated that ovarian cancer presents by non-specific symptoms like irritable bowel syndrome, fatigue and unexplained weight loss. ${ }^{14,15}$

Ultrasound is the primary diagnostic imaging tool of adnexal lesions \& the US ORADS structured terminology is used to accurately describe ovarian masses which lead to appropriate management strategies. ${ }^{16,17}$

Our U/S and color Doppler results of the studied 15 pre-menopause \& 35 post-menopause patients based on US-ORADS classification system revealed that:13 lesions showed benign criteria with US O-RADS score 3 while 37 lesions scored as US O-RADS 4-5 that considered to be likely of malignant etiology that was the most prevalent pathology in elderly postmenopausal patients. This was in coincidence with Zhang T 2017 who studied 263 masses, by U/S GIRADS and found that:- eighty sex were benign neoplasm (GI-RADS 3), one hundred and one were of GI-RADS 4 \& twenty eight were of GI-RADS 5 and cancer patients were older than patients with benign tumors. ${ }^{18}$ Also, AMOR FJ 2011 found that malignant tumors were more common in postmenopausal elderly women ${ }^{19}$ \& Li Zhou et al 2019 who retrospectively analyzed 224 patients with ovarian tumor and found that 120 were benign in patients aged $42 \pm 14.68$ years \& 104 were malignant in patients aged $53 \pm 12.73$ years. ${ }^{20}$ While, Mahmoud SA et al 2020 who studied 112 lesions using GIRADS classification concluded that: 36 lesions $(32.1 \%)$ were of GI-RADS 2, 32 lesions $(28.6 \%)$ were GI-RADS 3, 13 lesions $(11.6 \%)$ were GIRADS 4, and 31 lesions (27.7\%) were of GI-RADS 5 with $49 \%$ ovarian neoplastic lesions. ${ }^{21}$

Pre-operative diagnosis of ovarian lesions as benign or malignant very important for appropriate patient triage, referral, and management. ${ }^{22}$
According to our histopathology results for 39 lesions and final clinical diagnosis for 11 lesions:

Malignancy etiology was the most prevalent pathology " 70\%" while 15 lesions "30\%" were benign . Our results were in contrast to Zhang T et al 2017 results who studied 242 patients with 153 benign and 110 malignant tumors(18) \& Prasad1S et al 2019, who studied 56 masses and found 4 malignant masses, 24 benign masses and rest were physiological cyst/ infective process(22). While, Bhagde DA et al 2017, studied 50 patients with the majority below 45 years found that: All studied lesions were of benign etiology(13). Some authors stated that:-Ovarian fibroma can be misdiagnosed as it often exhibits features that are suggestive of malignancy. ${ }^{23,24}$

The essential role of O-RADS is to improve the quality and communication between interpreting and referring physicians, to guide patient management. ${ }^{25}$

Our study results using the U/S O-RADS score classification system in correlations to the final histopathology/clinical diagnosis revealed 11 true negatives, 32 true positives, 5 false-positive, and 2 lesions were false negative with $94.12 \%$, sensitivity, $68.75 \%$, specificity and $86 \%$ accuracy, $86.49 \%$ PPV \& 84.62 NPV that matched with Zhang T et al 2017 results which found 4 false-negative malignant cases that were misclassified as GI-RADS3, whereas 24 benign lesions with false-positive results that misdiagnosed as GI-RADS 4.

The sensitivity, specificity, false-positive, falsenegative \& accuracy, were $96.4 \%, 84.3 \%, 18.5 \%$, $3.0 \%, 89.3 \%$, respectively. ${ }^{18}$ However Mahmoud SA et al 2020 results revealed:- A diagnostic accuracy of US GI-RADS classification results were of $97 \%$ sensitivity, $73 \%$ specificity, $84 \%$ PPV, $94 \%$ NPV and $87 \%$ accuracy. ${ }^{21}$ While, Prasad 1 S et al 2019 concluded that: Excellent agreement between histopathology and U/S GI-RADS was found for the diagnosis of benign and malignant ovarian tumors with $100 \%$ sensitivity, $80 \%$ specificity, PPV $36 \%$, and NPV $100 \%{ }^{22} \&$ Li Zhou et al 2019 found that:The GI-RADS classification showed $99.1 \%$ Sensitivity, $85.9 \%$ specificity, $71.1 \%$ PPV and $99.6 \% \mathrm{NPV}^{20}$

\section{CONCLUSION}

The U/S O-RADS classification system was found to be a valuable non-invasive diagnostic tool of the suspicious ovarian masses with high sensitivity in differentiation between benign and malignant neoplastic lesions. 
This study had some limitations \& pitfalls due to the relatively low number of lesions included and as diagnosis of some lesions was relied on a follow-up clinical diagnosis.

\section{REFERENCES}

1. Givens V, Mitchell G, Harraway C et al. Diagnosis and Management of Adnexal Masses. Am Fam Physi-cian. 2009;80(8):815-22.

2. American College of Obstetricians and Gynecologists' Committee on Practice BulletinsGynecology. Practice Bulletin No. 174: evaluation and management of adnexal masses. Obstet Gynecol. 2016;128(5):e210-26.

3. Thomassin-Naggara I, Poncelet E, Jalaguier-Coudray A, et al. Ovarian-Adnexal Reporting Data System Magnetic Resonance Imaging (O-RADSMRI) Score for Risk Stratification of Sonographically Indeterminate Adnexal Masses. JAMA Network Open. 2020;3(1):e1919896 doi:10.1001/jamanetworkopen.2019.19896

4. Sayasneh A, Ekechi C, Ferrara L et al. The characteristic ultrasound features of specific types of ovarian pathology (Review). International Journal Of Oncology. 2015; 46: 445-58.

5. Jeong Y, K. Outwater E and Keun Kang H. From the RSNA Refresher Courses Imaging Evaluation of Ovarian Masses. RadioGraphics 2000; 20:144514701.

6. Sung Il Jung. Ultrasonography of ovarian masses using a pattern recognition approach. Ultrasonography. 2015; 34(3).

7. Dodge JE, Covens AL, Lacchetti C, et al. Management of a suspicious adnexal mass: a clinical practice guideline. Curr Oncol. 2012;19(4):e244-57. doi: $10.3747 /$ co. 19.980

8. Brown DL, Dudiak KM, Laing FC et al. Adnexal Masses: US Characterization and Reporting. Radiology: 2010; 254: 2.

9. Andreotti RF, Timmerman D, Strachowsk LM et al. O-RADS US Risk Stratification and Management System: A Consensus Guideline from the ACR Ovarian-Adnexal Reporting and Data System Committee. Radiology 2019; 00:1-18.

10. Andreotti RF, Timmerman D, Strachowski LM et al., O-RADS US Risk Stratification and Management System: A Consensus Guideline from the ACR Ovarian-Adnexal Reporting and Data System Committee. Radiology, 2020. 294(1): p. 168-85.

11. Menon U, Gentry AM, Hallett R et al. Sensitivity and specifi city of multimodal and ultrasound screening for ovarian cancer, and stage distribution of detected cancers: results of the prevalence screen of the UK Collaborative Trial of Ovarian Cancer Screening (UKCTOCS). Lancet Oncol 2009; 10: 327-40

12. Carvalho JP, Moretti-Marques R, Silva Filho AL. Adnexal mass: diagnosis and management. FEBRASGO POSITION STATEMENT. 12- 2020.

\section{DOI:https://doi.org/10.1055/s-0040-1715547}

13. Bhagde AD, Kamal SJ, Snehal MP et al. An analytical study of 50 women presenting with an adnexal mass. Int $J$ Reprod Contracept Obstet Gynecol. 2017;6(1):262-5.

14. Ebell MH, Culp MB, Radke TJ. A systematic review of symptoms for the diagnosis of ovarian cancer. $A m$ J Prev Med.2016;50(3):384-94 .

15. Goff BA, Mandel LS, Melancon CH, et al.. Frequency of symptoms of ovarian cancer in women presenting to primary care clinics. JAMA. 2004;291(22):2705-12.

16. Valentini AL, Gui B., Micc`o M., et al. Benign and Suspicious OvarianMasses-MR Imaging Criteria for Characterization: Pictorial Review. Journal of Oncology. 2012, Article ID 481806, 9. doi:10.1155/2012/481806

17. Margolies LR, Pandey G, Horowitz ER, et al. Breast imaging in the era of big data: structured reporting and data mining. AJR Am J Roentgenol 2016; 206:259-64

18. Zhang T, Li F, Liu J, et al. Diagnostic performance of the Gynecology Imaging Reporting and Data System for malignant adnexal masses. Int J Gynecol Obstet 2017; 137: 325-31.

19. Amor, FJ. Alica' Zar L, Vaccaro H, et al. GI-RADS reporting system for ultrasound evaluation of adnexal masses in clinical practice: a prospective multicenter study. Ultrasound Obstet Gynecol 2011; 38: 450-5.

20. Zhou L, Xuan Z, Wang Y. Diagnostic value of ultrasound score, color Doppler ultrasound RI and spiral CT for ovarian tumors. Oncology letters 2019; 17: 5499-5504.

21. Mahmoud SA, Talaat SH, Abd elghafar M, Egyptian Journal of Radiology and Nuclear Medicine. 2020; 51:60.

22. Prasad1 S, Kumar MJ, Sahu S et al. Evaluation of Ovarian Masses by Color Doppler Imaging and Histopathological Correlation. International Journal of Contemporary Medicine Surgery and Radiology $2019 ; 4,2$.

23. Timmerman D, Testa AC, Bourne T, et al. Logistic regression model to distinguish between the benign and malignant adnexal mass before surgery: A multicenter study by the International Ovarian Tumor Analysis Group. J Clin Oncol. 2005;23:8794-801.

24. Valentin L, Ameye L, Jurkovic D, et al. Which extrauterine pelvic masses are difficult to correctly classify as benign or malignant on the basis of ultrasound findings and is there a way of making a correct diagnosis? Ultrasound Obstet Gynecol. 2006;27:438-44.

25. Rochelle F A, Timmerman D, Beryl RB. OvarianAdnexal Reporting Lexicon for Ultrasound: A White Paper of the ACR Ovarian-Adnexal Reporting and Data System Committee, J Am Coll Radiol 2018;15:1415-29. 